\title{
Le programme Feederwatch et la politique des grands nombres
}

\section{Florian Charvolin}

\section{(2) OpenEdition}

\section{Journals}

Édition électronique

URL : http://journals.openedition.org/developpementdurable/687

DOI : 10.4000/developpementdurable.687

ISSN : 1772-9971

\section{Éditeur}

Association DD\&T

\section{Référence électronique}

Florian Charvolin, « Le programme Feederwatch et la politique des grands nombres », Développement durable et territoires [En ligne], Varia (2004-2010), mis en ligne le 19 juin 2004, consulté le 30 avril 2019. URL : http://journals.openedition.org/developpementdurable/687 ; DOI : 10.4000/

developpementdurable.687

Ce document a été généré automatiquement le 30 avril 2019.

\section{(cc) (i) \&)}

Développement Durable et Territoires est mis à disposition selon les termes de la licence Creative Commons Attribution - Pas d'Utilisation Commerciale 4.0 International. 


\title{
Le programme Feederwatch et la politique des grands nombres
}

\author{
Florian Charvolin
}

1 Le projet américain Feederwatch a pour but de compter les oiseaux aux mangeoires des particuliers de novembre à avril de chaque année depuis 1988. Ce programme s'appuie sur l'outil statistique pour dégager des tendances sur plusieurs années de l'évolution des populations et de leurs aires de distribution, dans ce qui s'apparente essentiellement à une recherche de dynamique des populations.

2 Pour bien prendre la mesure de ce qui se passe dans le programme Feederwatch, il faut alors faire d'emblée la distinction entre deux modes de constitution d'une population, comme peut l'être la population des oiseaux. Dans un cas on a un "collectif" d'oiseaux évoluant souvent ensemble ${ }^{1}$; leur identité de groupe est dépendante de la manière "ensemble" avec laquelle ils évoluent. On notera alors les comportements grégaires, les conflits territoriaux ou les stratégies de nidification etc. Dans l'autre cas, on a une "collection" d'entités dont on ne saisit l'identité que comme exemplaires d'une espèce. Cet exemplaire ne fait sens que comme exemplaire d'une série. Du "collectif" à la "collection" on substitue à la manière dont les oiseaux se coordonnent ensemble un autre type de qualité d'ensemble : l'appariement les uns aux autres d'exemplaires de la même espèce, rendant possible leur dénombrement ${ }^{2}$. L'apparition des oiseaux compte pour un dans un calcul d'absence/présence des oiseaux sur le site du comptage; toutes les occurrences accumulées les unes après les autres définissent des exemplaires de l'espèce suppositio distributiva (Descombes 2001, 321) : leur qualité d'espèce s'épuise dans tous ses exemplaires pris séparément les uns des autres (ou distributivement). Elle émerge indépendamment des structures sociales auxquelles les oiseaux seraient assujettis pour maintenir un certain état de leur identité. Cette vision diffère alors d'une définition de l'oiseau comme d'une entité qui n'autorise de parler d'un ensemble que par la manière collective dont se sont réunies ses composantes, comme la qualité du groupe innombrable de ses participants (et donc qu'il est vain de dénombrer). Dans ce dernier cas de figure en 
effet, la possibilité de parler d'un ensemble tient à la solidité de l'association entre oiseaux, collectivement.

Derrière ces deux possibilités de qualité d'ensemble, distributive ou collective se cache des philosophies différentes de la définition et du comportement des espèces d'oiseaux bien sûr, mais également de la position du centre de calcul qui recueille les données quantitatives face aux profanes qui les lui fournissent. Je vais montrer dans ce qui suit que le parti pris pour une logique distributive de la constitution des ensembles, associe une recension d'oiseau ordinaire conçue elle-même comme ordinaire et non spécialisée, dont l'exercice relève autant de la science que d'un modèle de comportement citoyen, conçu comme détenteur de bon sens et de savoir tacite, et par conséquent digne du rôle de témoin qu'on lui fait jouer. Cette assise sur la bonne volonté est au principe de la science ornithologique de Feederwatch (PFW); elle associe par conséquent bénévolat à programme scientifique, et dissout la frontière entre science et mouvement social. C'est alors que cette initiative prend son sens de science citoyenne, terme popularisé par les scientifiques du programme Feederwatch eux-mêmes dans les années 90, mais en parallèle aux travaux d'Alan Irwin sur le même sujet (Irwin 1995). Ce terme est dès lors quelque peu ambigu, selon qu'on se réfère à l'une ou l'autre des acceptions : d'un côté il convoque la sociologie critique cherchant à dégager de la vulgarisation scientifique (Public Understanding of Science or PUS), les comportements des citoyens qui en sont les destinataires. Plus de citoyenneté est alors le correctif à apporter à des programmes scientifiques défectueux. Est citoyen ce qui manque aux programmes scientifiques pour devenir de véritables programmes de PUS; de l'autre côté la science citoyenne désigne une réussite. Elle concerne des acquis des expériences passées. Elle juge de la démocratie en fonction de ses réussites locales qu'on pourrait généraliser. Cette vision de la science citoyenne rejoint alors les exemples de communauté thérapeutiques notamment (Epstein 1996, Callon, Lascoumes, Barthe 2001) qui envisagent la citoyenneté non pas comme l'antiprogramme de la science de laboratoire, mais comme des poches d'associations réussies entre profanes et scientifiques à étendre de manière incrémentale à la société.

Je réserverai à la discussion en fin d'article la question des implications socio-politiques de ce phénomène de science citoyenne qu'est l'activité de comptage des oiseaux. Je débuterai par l'étude de cas déclinée à partir d'abord du perceptible à l'échelle du profane, puis des corrections statistiques apportées à l'observation et enfin du genre de public créé par Feederwatch.

5 Le programme Feederwatch est lancé par le Laboratoire d'Ornithologie de Cornell (CLO) pour faire des coups de sonde sur tout le territoire des Etats-Unis et du Canada, et mesurer la population des oiseaux aux mangeoires ${ }^{3}$. Il regroupe 16000 profanes qui observent les oiseaux dans leur jardin. Tel que le programme est pensé, chaque oiseau observé est différent d'un autre en cela que les sites d'observations sont suffisamment dispersés pour qu'on puisse envisager qu'aucun oiseau ne passe d'un site à un autre. Il y a une chance infinitésimale pour qu'un oiseau observé sur un site soit le même que l'oiseau observé sur un autre site. D'autre part, ces oiseaux sont observés durant la période hivernale, largement en dehors des périodes de grandes migrations, ce qui favorise encore cet indigénat des oiseaux aux mangeoires des profanes. Ceux qui sont observés ne bougent que faiblement sur toute la période de novembre à avril. Ils ont un comportement influencé par l'offre de nourriture et d'eau qui leur est faite par les profanes, qui fait dépendre le regroupement de cette fraction du total de la population aviaire de son assiduité aux mangeoires. Ils sont comptabilisables parce qu'ils se prêtent à 
l'observation rapprochée en fonction d'un centre d'attraction propre: la mangeoire, dans le cadre d'observation du jardin, sous l'oeil du profane. Le protocole d'observation du CLO stipule bien que ces oiseaux, pour être comptés, doivent entretenir une relation d'attachement avec le lieu, qu'il s'agisse de l'attraction de la nourriture, de l'eau, du bain ou bien la prédation, par les rapaces, des oiseaux assidus aux mangeoires. Le profane ne doit pas compter les oiseaux qui ne font que survoler le site, pour que ceux qui sont retenus ne relèvent que d'une dépendance au site et ainsi correspondent à un lieu et un seul, ce qui est la règle imposée par Cornell ${ }^{4}$.

6 Il est important que l'oiseau soit vu selon une occurrence unique, dans un seul lieu et dans un seul moment, pour obtenir des variables mutuellement exclusives et donc discrètes. On demande au profane d'épurer de son observation les éléments qui pourraient enchâsser les oiseaux dans des dynamiques collectives sur le site de comptage telles que les comportements territoriaux ou les stratégies d'accouplement, etc.; le protocole d'observation diffusé par le CLO insiste pour que le profane se limite à la mesure en terme de décompte de présence/absence et de nombre des oiseaux vus aux mangeoires. Les mesures sont discrètes. On ne demande pas d'observer des oiseaux dont le collectif découlerait d'une édification passée ensemble (comme les comportements grégaires, la construction de nids ou les parades nuptiales), mais des unités discrètes, agrégeables distributivement, des électrons libres dont l'impossibilité d'assignation à un "faire ensemble" qui définirait leur relation, permet d'établir leur rapport sous leur plus petit commun dénominateur à savoir leur addition et finalement leur collection. Nombres d'oiseaux aux mangeoires, distribution et mouvements de population sont les trois catégories d'analyse que Bonney et Dhondt disent être visées par les biologistes qui écrivent les résultats préliminaires du programme dans la revue du CLO: Birdscope (Bonney et Dhondt, 1997, 43). Rien de la dynamique collective notée aux mangeoires ne doit interférer avec l'enregistrement purement distributif de la population aviaire. Et l'accent est mis sur l'exhaustivité avec laquelle cet enregistrement doit être fait, nonobstant le caractère plus ou moins trivial de l'observation. C'est pour cette raison qu'on demande aux profanes de bien noter lorsqu'ils ne voient pas d'oiseaux (ce qui est impliqué par le fait que le protocole demande aux profanes de noter ce qu' ils voient ou ne voient pas pendant des jours choisis au préalable comme jours d'observation) car cette donnée négative rentre comme élément du décompte et servira à dresser les cartes de répartition des espèces; cela va cependant à l'encontre de leur tendance à ne reporter sur leur fiches d'observation que les occurrences positives d'oiseaux, et singulièrement les fois où des oiseaux exceptionnels sont vus.

7 Donc les profanes sont considérés comme des relais de vision, des capteurs dotés d'une capacité à s'en tenir à ce qu'ils voient, certes à rien d'autre que ce qu'ils voient, mais aussi à tout ce qu'ils voient c'est-à-dire aussi des observations bizarres. Cela peut introduire un doute sur la pertinence de l'observation. L'oiseau est-il bien nommé par le profane? N'y a-t-il pas fraude sur l'observation? On accorde au profane la possibilité de fournir des jugements compétents, sans qu'il ne soit possible au centre de calcul de Cornell de contrôler la compétence de chacun en fonction de ses états de service ornithologiques ${ }^{5}$. Le problème se pose bien évidemment avec beaucoup d'acuité pour les observations inattendues. La recherche de la collection d'oiseaux plutôt que du collectif ménage alors une issue à ce problème. Toutes les informations transmises par les profanes sont colligées et dégagent pour la plupart des écarts les unes d'avec les autres -ce qui est compréhensible étant donné que le programme est réputé ne mesurer à chaque fois que 
des oiseaux différents les uns des autres, de manière mutuellement exclusive-, certes, mais selon des rapports dotés d'une certaine proportionnalité. Ce qui compte n'est pas le chiffre isolé mais la position du chiffre relativement aux autres chiffres collectés. On cherche à dégager des tendances et des variations, ce qui suppose d'avoir plusieurs points -quand ce n'est pas un maximum- de référence. Ce qui compte c'est la relation entre chiffres qui seule peut montrer des tendances temporelles ou des différences de distribution spatiale, laissant tomber l'importance du chiffre en soi. Qu'importe la validité du chiffre en soi, et l'éventuelle erreur qui s'y glisserait, si la variation des chiffres les uns relativement aux autres est significative. Ainsi pour calculer le degré de correspondance entre les données issues du dispositif d'observation Feederwatch et ce qui se passe dans la nature, on a recours non pas aux chiffres en soi mais aux variations issues de comparaisons soit spatiales soit temporelles. Lepage et Francis (Lepage et Francis 2002) montrent que pour savoir si Feederwatch mesure bien ce qui se passe dans la nature, il faut comparer à Feederwatch plusieurs dispositifs d'observations comme le Christmas Bird Count ou le Breeding Bird Survey, et ceci n'est possible que s'il y a des variations dans chacun des programmes, qu'il est possible de corréler d'un dispositif à l'autre pour constater la plus ou moins grande congruence de l'un à l'autre. Comme c'est souvent le cas dans l'observation de terrain, si «ça bouge », c'est visible et donc appréhensible. S'il n'y a pas de variation dans les données d'une année sur l'autre, il n'y a pas de corrélation entre les indices du Christmas Bird Count de Feederwatch ou du Breeding Bird Survey, et il n'y a par conséquent pas moyen de savoir si l'observation faite correspond à un état de la population dans la nature. Cela suppose en effet d'avoir un moyen, lorsqu'on descend en deçà d'un seuil quantitatif d'occurrences d'oiseaux, d'être capable de discriminer la faible variation du bruit de fonds introduit par l'erreur de jugement ou l'inégale répartition des profanes sur tout le territoire nord-américain. On va voir maintenant que ces bruits de peuvent être corrigés statistiquement.

8 Le problème majeur auquel est confronté le statisticien du CLO est de distinguer le bruit de fonds, qui est l'impondérable d'un dispositif d'observation et peut n'être pas gênant outre mesure si le ratio entre lui et le signal passant dans le dispositif est suffisant, et le biais qui suppose non pas l'erreur du hasard mais une faiblesse systématique dans le dispositif de comptage (entretien avec Wes Hochachka). On peut corriger le bruit de fonds introduit par des informations bizarres en provenance des profanes, sans que leur erreur de jugement, remette en cause l'ensemble du dispositif. Il suffit de savoir que cette marge d'erreur est constante et mesurable, permettant de produire des résultats avec un intervalle de confiance constant d'une mesure sur l'autre, ce qui n'altère pas le calcul des tendances sur plusieurs années ou sur plusieurs aires géographiques ${ }^{6}$. De plus, en raisonnant sur les grands nombres, on sait qu'à partir d'une certaine quantité d'information engrangée sur la même base, la représentativité est calculable statistiquement indépendamment de chaque observation prise séparément.

Pour finir de gommer tout biais dans la collecte des données, il faut éliminer au maximum l'éventuelle erreur introduite dans la machine statistique par la première observation. Après ce stade d'observation de terrain, en effet, le modèle pourra tourner tout seul et, pourvu que les algorithmes soient bons, il y aura pertinence de la donnée obtenue. C'est ainsi par exemple que le programme PFW se corrige automatiquement d'une année sur l'autre. En effet la liste répertoriant les oiseaux observables qui est transmise à chaque profane est basée sur les résultats statistiques de l'année précédente et non pas sur une 
connaissance infuse de ce qui est susceptible d'être observé aux mangeoires. Le système tourne en vase clos.

10 Il est alors de la plus haute importance d'éliminer au maximum l'influence de l'étape initiale, celle qui a fourni arbitrairement la première liste des oiseaux à partir d'observations visuelles. De ce point de vue, le modèle conçu n'est pas déterministe mais probabiliste. Il n'est pas déterministe en ceci que les données de l'observation au temps Tn ne sont pas celles qui déterminent directement et sans modification les résultats en terme de tendance ou d'aire de distribution des oiseaux au temps $\mathrm{Tn}+1$. Non; le statisticien reconnaît à chaque observation un degré d'incertitude et lui affecte un algorithme en fonction d'une probabilité d'apparition assise sur les tendances extrapolées des années précédentes. Si on prend en compte le hasard, quelle chance d'observer tel oiseau a le profane? Telle est la question posée par le statisticien. De cette manière, on échappe au modèle déterministe fixe, pour raisonner sur un modèle probabiliste, où l'observation initiale de terrain, l'étape un du modèle est "oubliée » par le jeu probabiliste. Très rapidement, après quelques années de campagnes, on a délaissé l'observation directe et de terrain, pour l'expérience modélisatrice.

11 Le statisticien raisonne sur la possibilité de tirer au sort n'importe quelle donnée comme aune à laquelle mesurer le caractère informatif ou pas de la donnée. En conséquence, la donnée ainsi obtenue est plus proche de ce que les profanes ont des chances de voir aux mangeoires, que si on cherchait à faire simplement la moyenne arithmétique en divisant la somme des observations par le nombre d'observateurs ${ }^{7}$. Cette introduction du hasard met sur un pied d'égalité tout un chacun devant les impondérables de l'observation. Cela permet, en outre, une égalité plus grande de traitement de chacun des observateurs qui n'a pas plus de poids qu'un autre en face du hasard du tirage au sort de telle ou telle donnée.

12 Ce faisant le calcul statistique se rapproche plus de ce que tout un chacun a des chances de voir aux mangeoires nivelant les observations non pas en fonction de ce que les plus aptes ou les plus chanceux sont capables de voir, mais en fonction du hasard. C'est parce qu'il fait fonds sur le hasard de la distribution des espèces, exprimable à partir d'une certaine quantité d'information collectée, que le chiffre obtenu n'est plus lié aux aléas de l'observation, dépendant du comportement plus ou moins collectif des oiseaux aux mangeoires ou de la perspicacité plus ou moins grande des observateurs. Il est lié à une tendance quantitativement exprimée et significative pour autant que le ratio entre signal (tendance notoire) et bruit de fonds (aléas de l'observation) le permet. Au delà d'un certain seuil de collecte de données, fournies en fonction du suivi d'un protocole, le chiffre a une représentativité qui n'est plus affectée par les erreurs de tel ou tel observateur. Ainsi la statistique, si elle peut obtenir des grands nombres, c'est à dire si elle peut se baser sur de nombreux profanes, permet de se passer d'une sélectivité dans le choix des observateurs en fonction de leur compétences. Grâce à la statistique, on substitue à la recherche de la compétence de l'amateur, l'effet de masse issu du recrutement de nombreux profanes, qui permet d'atteindre des niveaux de signification statistique des données. Le programme mise sur une absence de barrière à l'entrée (entretien avec Rohrbaugh ${ }^{8}$ ) de manière à ce que le maximum de personnes y participent, produisant des données quantitatives et donc correctrices de l'erreur d'observation plus grande que ces profanes occasionneront faute de formation préalable.

13 Cela signifie que le dispositif de Feederwatch devient sensible pour les données non orphelines, c'est à dire à partir du moment où des conséquences indirectes de 
l'observation des oiseaux par des profanes les unes sur les autres adviennent. Ce n'est qu'en présence du caractère indirect du comptage qu'une observation, par le détour par d'autres observations, peut être reconnue. C'est pour cette raison notamment que la rareté est calculée comme une fonction de la temporalité -l'observation est comparée à d'autres observations antérieures ou postérieures- et d'aire de distribution de l'espècel'observation est comparée à d'autres observations dans des lieux voisins ou distants-. Ce qui fait qu'un privilège est donné aux observations non orphelines, c'est que ces données sont plus robustes une fois pris en compte le fait qu'elles sont totalement indépendantes les unes des autres; donc deux observations identiques, sous couvert d'un voile d'ignorance entre observateurs, signifient une plus grande chance d'occurrence de l'oiseau. La distance géographique de fait entre profanes ou sites de comptage, sert de voile d'ignorance entre eux, permettant à une donnée répertoriée dans plusieurs lieux d'être plus robuste que s'il avait fallu compter avec des sites de comptage enchâssés les uns dans les autres, ou des profanes familiers les uns avec les autres'. C'est le prix à payer pour que la population soit déductible de l'échantillon prélevé : «Cette implication prend d'habitude la forme d'une implication à propos de l'échantillonnage au hasard de la population ou bien elle induit que les mesures sont indépendantes et issues d'une distribution probabiliste qui est la même que celle de la population» (James et McCulloch, 1984, 29). On est sûr, avec ce système, que le décompte s'est fait au hasard, c'est-à-dire à l'aveugle, sans coalitions entre les oiseaux ou même entre les profanes. Le voile d'ignorance est le garant qu'on est en présence d'entités dont la capacité à la coalescence apparait purement sur le mode de la collection et non d'un "faire ensemble". Les oiseaux font nombres et ils ne font que cela. Dès lors, comme on l'a vu, pour des raisons de validation statistiques, on préfère les observations faites en nombres par rapport aux observations plus rares. Ce privilège donné à l'observation pléthorique va dans le sens d'un privilège donné à l'oiseau commun.

Il est inutile d'insister plus avant pour tirer les conséquences de cette petite étude d'une politique de la collection des observations d'oiseaux. Dans la discussion qui suit, je relève quelques traits caractéristiques qui pourraient s'appliquer à d'autres opérations de comptage impliquant des amateurs, dans le champ des sciences naturalistes ou éventuellement des sciences sociales.

On est en présence d'un schéma atomiste du groupe des oiseaux, certes saisis en tant qu'ils font population, mais pas dans leur structuration passée collectivement, comme le veut le "totus" latin (tels les comportements territoriaux ou les stratégies $\mathrm{d}$ 'accouplement), mais selon une logique purement distributive et démographique de leur existence, selon le "omnis" latin. Cette distinction entre le collectif et le distributif est rapporté par Vincent Descombes à propos de la logique : "Ce qui est blanc, est-ce les choses blanches prises ensemble, donc leur ensemble (comme on dirait l'ensemble innombrable des choses blanches) ? Est-ce que ce n'est pas plutôt chacune des choses blanches, donc toutes les choses blanches prises une à une ("distributivement et non "collectivement" selon la distinction classique ) ?" (Descombes 2001, 316). Les oiseaux sont un bon cas d'étude de cet appariement distributif car le programme se préoccupe de préférence de leur "distribution" dans des aires distinctes ${ }^{10}$. On insiste sur le caractère unique de l'oiseau observé dans la singularité duquel se reconnaît le caractère de l'espèce. D'où l'importance du décompte d'occurrences sans autre rapport les unes avec les autres que leur appartenance à la même catégorie: des cas "d'espèce "; d'où l'importance d'occurrences mutuellement exclusives comme on l'a vu au début de ce texte ${ }^{11}$. De même, 
dans le cas d'observation d'oiseaux rares, on demande les informations suivantes au profane: qui, quoi, où, quand (entretien avec Laura Kammermeier) pour bien documenter le caractère mutuellement exclusif des données, s'assurant qu'on est en présence d'un et d'un seul élément de vision à l'exclusion de tous les autres, à chaque fois.

Avec l'aire de distribution, qui est, avec la tendance annuelle, le principal produit final visé par Feederwatch, le CLO insiste également sur l'importance de la donnée " ponctuelle». Puisque qu'on obtient pour chaque campagne de mesure que des points, l'aire de distribution dont je parle ne peut apparaître que comme un piquetage du territoire et non un report sur carte de zones de présence. En effet pour produire une aire il faut passer du point à l'étendu et cela nécessite un mécanisme appelé interpolation. Ces aires sont interpolées par le spécialiste des $\mathrm{SIG}^{12}$. Cela veut dire qu'une surface sur la carte ne correspond pas exactement à une concentration de points d'observation, car si cela était le cas, on aurait des points et non des surfaces. Pour obtenir des surfaces il faut savoir à tout moment le rapport que tout point de la carte entretient avec ses voisins plus ou moins éloignés et pas seulement se limiter à connaître les seuls points d'occurrence des oiseaux. Pour calculer des surfaces, le spécialiste des SIG calcule la plus petite distance existant entre tout point de la carte et un site d'observation où a été signalée telle espèce. Il en tire un ratio qui lui permet de fixer une échelle de signification pour chaque point de la carte : en deçà d'un certain seuil un point de la carte ne sera pas grisé pour donner une surface, ou bien au delà de ce seuil ce point sera grisé et rentrera dans une aire de présence de l'oiseau. Ce procédé pour répartir les observations géographiquement est l'échelon le plus évolué et le plus évident du caractère indirect, de la signification de l'observation d'un profane, en fonction du détour par les autres observations, puisque tout point de la carte obtient une signification géographique uniquement en fonction de sa relation aux autres points.

On peut résumer alors plusieurs éléments abordés au cours de cet article pour montrer l'avantage d'une conception quasi-épidémiologique de l'évolution et la distribution des oiseaux, qui sied à une position d'expertise neutre et capable de faire des pronostics. Le recours à un modèle probabiliste rend en effet capable de dresser des pronostics sur l'ensemble de la population d'oiseaux de manière plus souple qu'un modèle déterministe qui buterait sur le fait qu'on travaille sur de faibles échantillons et qui plus est sur des oiseaux semi-domestiqués. L'assise sur le hasard -si on tire au hasard quelle est la probabilité d'obtenir telle donnée- garantit un flexibilité explicative en terme de chance d'apparition et non de certitude d'apparition. Au niveau du modèle statistique les oiseaux n'ont que des chances d'apparition. Mais du coup, ce savoir est plus généralisable et robuste par rapport aux attaques qu'on pourrait lui faire -comme l'inégale répartition des observateurs sur tout le territoire, etc. Si on dispose de populations statistiquement représentatives, on peut corriger ce type de bruit de fonds. De la même manière le calcul des étendues par le spécialiste des SIG, consiste à rendre relatif à l'ensemble des observations la position d'un point sur la carte. Une zone grisée représente une zone à "chance " d'observer telle espèce mais non pas une zone d'apparition effective. Là encore, ce qui compte c'est la capacité de prévision de l'occurrence des oiseaux pour celui qui lit les cartes interpolées. Le lecteur sait où il y a des chances d'observer l'oiseau quel que soit la zone qu'il regarde, illustrant que «la nature ne fait pas de sauts». Depuis ce point de vue, les zones interstitielles entre des points d'occurrence ne sont pas des zones de non savoir -on ne sait pas ce qu'il y a entre les points d'occurrences- mais des zones de plus ou moins grande chance d'observation de l'espèce. En lisant ces cartes, on est doté de 
capacités de pronostics, ce qui renvoie à l'optimisme scientifique à l'œuvre au Laboratoire d'ornithologie de Cornell et à sa tendance à faire de la prescription et du conseil auprès des autorités et du public ${ }^{13}$. On est alors dans un cas de figure proche du sondage -comme peuvent l'être les sondages d'opinion; se combinent une prévisibilité des données propre à corriger le caractère profane de leur extraction, et un anonymat obtenu non pas par une observation objective mais par une écriture à plusieurs mains.

est le plus important dans ce relevé de points d'observations c'est de pouvoir compter, c'est à dire non pas d'avoir des éléments dissemblables (des oranges et des navets) mais des séries homogènes parallèles pour chaque espèce. Il faut réduire la complexité de ce qui est observé à un format aplati et facilement quantifiable, à savoir des colonnes par espèce et des chiffres dans chaque colonne. Ce qui compte le plus dans cette politique quantitative c'est l'expression du nombre par espèce, étant entendu que plus le nombre est important plus le résultat sera bon. Il faut des grands nombres nous explique le statisticien. Cela favorise alors l'oiseau commun ${ }^{14}$. C'est cet oiseau pour lequel on a le moins de 0 réponse, qui est privilégié dans les analyses statistiques ${ }^{15}$. Dès lors, on saisit l'association opérée par le CLO entre une nécessité statistique -avoir le plus possibles d'observations positives- et la préoccupation du profane- voir le plus d'oiseaux possible. Le calcul probabiliste des chances de voir des oiseaux est à l'interface du savant, pour lequel la probabilité sert à estimer ce qui se passe dans la nature à partir d'un faible échantillon, et du profane, pour lequel la probabilité guide les attentes, voire les déplacements dans la quête de l'oiseau.

Mais il y a plus. Les oiseaux ordinaires sont ceux qu'un observateur a des chances de voir durant l'hiver à ses mangeoires (la likeliness du statisticien), ce qui réduit d'autant l'irrationalité potentielle d'un comportement de profane sur lequel Cornell n'a pas d'autre prise, puisqu'on peut anticiper en période de calme, les tendances de ce qui sera vu aux mangeoires d'une année sur l'autre. Comme on l'a dit le formulaire de saisie des données envoyé par Cornell ou accessible en ligne liste uniquement les oiseaux les plus susceptibles d'être vus aux mangeoires. Il " prémache » le travail pour les profanes, pour leur faciliter la tâche mais également pour rendre leur comportement prédictible. Lors de la réception par le CLO des formulaires une fois remplis, il n'y aura pratiquement plus que des listes préencodées d'oiseaux significatifs statistiquement.

Cette apparence de neutralité est en fait une coercition douce qui restaure une dimension politique à l'exercice de la science ${ }^{16}$. On a vu comment le travail du profane était " prémaché » et contrôlé à de multiples échelles, selon des modalités, qui, à l'instar des guides d'observation d'oiseau servent de guides et établissent un pouvoir de suivi entre les différentes étapes de la collection des données. On touche ici ni plus ni moins à la dimension rhétorique de la politique du CLO pour convaincre. L'anonymat qui ressort $\mathrm{du}$ comportement faussement machinal des profanes est le faire valoir du CLO pour prouver que ses données sont neutres et donc scientifiques. Mais cet anonymat l'est en conséquence de l'écriture à plusieurs mains qui a occasionné la circulation des informations sous la forme des fiches suiveuses et des formulaires remplis sur internet. Le CLO place son effort de recherche sur la dissociation de la donnée d'avec la subjectivité de l'observateur: il neutralise la singularité de chaque personne en ayant recours au correctif apporté par les grands nombres. Cette neutralisation est obtenue de haute lutte en conséquence de la massification du réseau d'observateurs -on met tout dans le pot commun- mais en même temps le souci de la traçabilité des données aux différentes phases de la saisine, l'indexation et le traitement des documents. Si les noms des profanes 
disparaissent dans l'opération de traitement des données par le CLO, en revanche l'indexicalité qui les a fait être des points locaux, circonstanciels et contextuels de contact avec tel oiseau et pas tel autre, reste comme le meilleur garant scientifique qu'on peut parcourir la chaîne d'inscription statistique des données dans les deux sens, du site d'observation au résultat statistique et vice versa ${ }^{17}$.

En effet, ce qui garantit la force probabiliste des modèles empiriques développées par le CLO c'est le contrat tacite passé entre le laboratoire et les profanes. Il sert à remettre à un tiers institutionnel, celui sous les auspices duquel peut être déployée la nécessaire bonne entente comme garant de la communication verbale dans nos sociétés modernes, le soin de garantir la loyauté des partenaires dans le témoignage que chaque profane fait à partir de la population vue à ses mangeoires (Dulong 1998). En dehors de la bonne volonté des bénévoles il n'y a pas d'autre dispositif que le respect de la pétition de principe permettant la communication, garantissant que les données transmises ne sont pas totalement inventées. C'est sur cette dimension tacite que repose le programme PFW, et finalement l'incontournable amateur.

Certes le CLO privilégie l'observation machinale en cela qu'elle est toujours la même et qu'elle est commune à des personnes desquelles elle ne requiert qu'une formation minimale. Toutefois c'est en raison même du caractère ordinaire de cette observation qu'on fait appel à du savoir tacite. On sait que contrairement au savoir et aux techniques fortement spécialisés et formalisés, l'ordinaire de la compétence technicienne est ce qu'on peut difficilement délocaliser tant elle va de soi dans un certain contexte. On ne la voit pas dans son contexte et donc dès lors qu'on la sort de ce dernier on est démuni devant le fait qu'elle ne fonctionne plus. Les points d'appui contextuels, comme les indices remarquables, servent de trucs pratiques pour nommer les oiseaux en situation. C'est cette action de typification qui est reconnue comme compétence aux amateurs, c'est à dire comme une compétence contextuelle, in situ, locale et instinctive. On s'en convaincra en constatant combien l'observation passe par l'abréviation des étapes de reconnaissance rendue possible par un modèle à grand trait (souvent la représentation des oiseaux dans les guides est graphique et non photographique), permettant d'aller à l'essentiel au moment, toujours furtif de la reconnaissance. De même le dépliant envoyé avec le protocole liste les principales difficultés de reconnaissance d'espèces voisines, ce qui va dans le sens d'une capacité de typification des oiseaux dévolue au profane. Dans le savoir mobilisé pour traiter une chaîne d'étapes de reconnaissances, l'abréviation, les trucs -les indices remarquables par exemple-, sont autant d'éléments instinctifs qui supposent un savoir tacite non formalisable par une machine (Charvolin, 2001). Sans le profane et sa compétence, il manque le va-et-vient entre pratique de terrain et classification ou évolutionnisme théorique, et l'ornithologie, en tant que science de plein air, n'est plus nourrie. La division ne passe pas entre des profanes collecteurs de données brutes et des scientifiques confinés en laboratoire. Il est loisible de montrer que les profanes aussi manipulent la théorie et il existe un aspect participatif à des programmes scientifiques comme Feederwatch, dans lequel le profane contribue à l'exercice de la science (Trumbull et al. 2000).

Ce qui compte alors c'est la démarcation entre expérience authentique et inauthentique de l'activité scientifique. Une des raisons les plus récurrentes pour laquelle les participants à Feederwatch adhèrent est qu'il s'agit de "science réelle » et non pas d'exercices propédeutique à visée uniquement pédagogique. On rejoint alors ce que Roth 
et Désautels appellent les expériences authentiques qui découlent de personnes au comportement du même nom (Roth et Désautels, 2004).

Il faut entendre par là des personnes en prise avec la réalité, adoptant une posture réaliste qui correspond assez nettement à la vision du citoyen dans le modèle d'Irwin et dans celui de Wynne (Irwin, 1995 ; Wynne, 1996). Il s'agit dans leur modèle toutefois de citoyens réticents, dont l'apparente apathie devant les programmes de vulgarisation scientifique n'est pas due à l'absence de culture scientifique (scientific literacy) mais d'un excès de compréhension de l'importance de ce qui se joue dans les sciences et techniques, qui les amènent à juger de la légitimité des vulgarisateurs en même que de l'authenticité des savoirs. Les profanes ne sont pas plus ignorants de «la » science qu'un physicien des particules ne connaît la chronaxie musculaire. Mais cependant ils partagent une vue réaliste qui fait qu'ils ne sont pas prêts à adhérer à un discours de vulgarisation sans examiner les états de services des personnes qui sont derrière ce discours. On trouve ici une première définition de la citoyenneté scientifique. Mais elle est encore trop négative pour le cas en présence, puisque dans le cas de Feederwatch il s'agit de "public understanding of science » réussi.

Bien évidemment la posture critique du citoyen «négatif» comme on pourrait l'appeler est retournée en enthousiasme à participer par les membres du projet Feederwatch. Il faut y voir une proclamation d'anti-utilitarisme dans les professions de foi des amateurs (Allen, 1994, 65) qui pointe dans la direction d'une morale du désintéressement ; c'est ce qu'il faut entendre aussi par l'enthousiasme à participer dans le programme Feederwatch et son attractivité pour des milliers de profanes. Ne faut-il pas y voir une des caractéristiques de la sociabilité nord-américaine, fondée sur l'importance de la participation dans des corps intermédiaires -autre définition du citoyen comme personne engagée- mais sur des bases pragmatiques et réalistes (Bellah, 1996), d'où l'attractivité de la science ? La rigueur du protocole de Feederwatch anticipant la rigueur de l'observation n'est-elle pas cette pétition de principe selon laquelle tout le monde doit jouer le jeu de l'investissement authentique dans le programme comme modus operandi de la vraie science, celle qui, pour reprendre Allen, s'invente une visée morale -citoyenne-dans un monde dominé par l'utilitarisme, et une mise en application collective -comme on a pu le voir-dans un monde dominé par l'individualisme?

Allen D.E. (1994), The naturalist in Britain, Princeton, Princeton University Press. 270 p.

\section{BIBLIOGRAPHIE}

Bellah, R. N. et al. (1996), Habits of the heart, Berkeley, University of California Press, 355 p.

Bonney R., Dhondt A. A., (1997). Feederwatch, an example of a student-scientist partnership, Cohen K. C. (ed.). Internet links for science education : student-science partnerships, New York, Plenum, pp.31-53.

Callon M., Lascoumes P., Barthe, Y. (2001). Agir dans un monde incertain, Paris, Seuil, 358 p. 
Charvolin F. (2001), Action à distance et engagement au Comité Ornithologique Rhône-Alpes, Ion J. (ed). L'engagement au pluriel, Saint-Etienne, Presses Universitaires de Saint-Etienne, pp.135-159.

Descombes V., (2001), Les individus collectifs, Revue du Mauss, 18, pp. 305-337.

Dhondt A.A., Tessaglia D.L., Slothower R.L., (1998), Epidemic mycoplasmal conjunctivitis in house finches from eastern north America, Journal of Wildlife Disease, 34/2, pp.265-280.

Dulong R. (1998), Le témoin oculaire, Paris, Presses de l'EHESS, 237 p.

Epstein S. Impure Science, Berkeley, University of California Press, 466 p.

Farber P.L. (1997), Discovering birds, Baltimore, John Hopkins Univ. Press.

Irwin Alan. (1995), Citizen science, London, Routledge,198 p.

James D. McCulloch C. (1984). Data analysis and the design of experiments in ornithology, Current Ornithology, n.2, pp.1-63.

Lepage D., Francis C.D., (2002), Do feeder counts reliably indicate bird population changes ? 21 years of winter bird counts in Ontario, Canada., The Condor, 104, pp.255-270.

Rémy, E., (1995), L'élaboration d'un savoir naturaliste. Approche anthropologique d'un atlas, Etudes rurales, janvier-mars 1995, 137 : 55-72.

Roth W-M, Désautels J., (2004), Educating for Citizenship : Reappraising the Role of Science Education, unpublished paper, avril.

Trumbull D.J., Bonney R., Bascom D.. Cabal A., (2000), Thinking scientifically during participation in a citizen-science project, Science Education, 84, pp.265-275.

Wells J.F., Rosenberg K.V., Dunn E.H., Tessaglia-Hymes D. L., Dhondt A.A., (1997), Feeder counts as indicators of spatial and temporal variation in winter abundance of resident birds. Journal of Field Ornithology, 69/4, pp.577-586.

Wynne B. (1996) May the sheep safely graze ? A reflexive view of the expert-lay knowledge divide, in Wynne B., et al. Risk, Environment and Modernity, Thousand Oaks, Sage, pp.44-83.

\section{NOTES DE FIN}

1. En ce sens qu'ils participent d'écosystèmes les mettant en relation constructive avec d'autres espèces.

2. La question du dénombrement des espèces et donc de leur nomenclature est une question récurrente de la littérature ornithologique depuis le XVIII ${ }^{\mathrm{e}}$ siècle. Elle s'oppose à la question plus récente de leur éthologie, et vient d'une période de l'histoire de l'ornithologie où il fallait faire un effort de classement devant l'énorme quantité d'information qui provenait des naturalistes voyageurs (Farber 1997, chap.III). 3. Le site internet du programme est le suivant : http://www.birds.cornell.edu/pfw/ 4. D'où la précaution avec laquelle les membres du CLO extrapolent la population totale à partir de la population vue aux mangeoires. Les oiseaux observés peuvent donner une image tronquée de la population totale du site, si par exemple les mangeoires attirent plus les individus faibles ou malades résultant en une surreprésentaton de certaines fractions de la population aviaire, qui doit être corrigée par la statistique (Dhondt et al. 1998, 268). C'est ainsi qu'on ne peut pas prédire sans évaluation poussée préalable,si les 
observations faites aux mangeoires sont representatives de la population d'oiseaux dans la nature (Wells et al., 1997).

5. Contrairement a ce qui se passe avec les personnes relais dans le cas de la mise en place d'un atlas herpétologique. (Rémy, 1995).

6. C'est ce que Lepage et Francis appellent des "reliable standard errors" (Lepage et Francis, 2002, p.258).

7. La courbe du nombre d'observations rapportée au nombre d'observateurs n'est en effet pas linéaire, ce qui correspondrait à une moyenne arithmétique, mais elle est courbe, c'est à dire plus haute que la moyenne pour la grande fraction des profanes qui observent peu d'oiseaux de la même espèce aux mangeoires, et plus basse que la moyenne pour la faible fraction des profanes observant de nombreux oiseaux de la même espèce aux mangeoires. La chance d'observation n'est pas calculée de manière arithmétique, mais en fonction du tirage au hasard, c'est à dire de la valeur la plus proche de ce qu'on obtient comme donnée si on tire au hasard. Le statisticien du CLO nous explique: "If you're to go at random to a feederwatcher in some region, what is the likely number of birds they would see. What is the likely number of chickadees or robin or grosbeaks or whatever it is. And intuitively the likely number or the number typically seen is better approximated by a geometric mean. If you are to do a histogram showing the number of participants on the vertical axis and then on the horizontal axis the number of individual birds observed of a species, the distribution is not a normal distribution so it doesn 't have a few people seeing very few birds most people seeing an intermediate number of birds and very few people seeing a high number of birds. Instead what you usually see is that most people see few individual birds and then you have some people who have exceptionally large numbers of individuals. And with the geometric mean the way it produces a summary value that's closer to what the high peak of the histogramm is (...). When you transform you are essentially bringing that tail in much closer, you are giving it less importance, so that your summary statistic more closely reflects what that peak number of individual observers are seeing."

8. "We don't rely to National Audubon Society (NAS) at all to filter their participants or their members to produce a more reliable high quality participant process in science protect that is going to provide top knowledge biological data. And we don't make the assumption that just because someone is a member of the NAS that he knows more as other participants. I think that this would be a very wrong assumption. There are lot of people who are members of the NAS that are not necessarily inclined towards science or birders that really know the birds extremely well [and are not member of the NAS]. Instead we don't try to filter or preselect the people that participate in citizen science projects, which would be virtually impossible, and also which would preclude us from completing one of our objectives which is to educate. If we were preselecting our audiences there would only be involving a small segment of the population and we would not be educating these other folks. So instead of doing that we go to various procedures that allow us to break protocols, design data forms and in general ask appropriate questions that the layperson can answer".

9. Il est frappant de constater également que ce fonctionnement en aveugle de la collecte des données est conforté par le mode de recrutement des profanes par voie de presse essentiellement. Bonney et Dhondt expliquent que " les participants sont sollicités dans le groupe des members du CLO et dans le public général par lettre directe, publicité, annonces et mailing ciblés aux clubs ornithologiques et aux chapîtres de la Société nationale Audubon" (Bonney Dhondt, 1997, p.33). 
10. C'est ainsi que pour certaines sous-espèces voisines dans la classification, c'est essentiellement la localisation dans telle ou telle partie du continent américain qui présidera à leur rangement dans telle ou telle catégorie.

11. Il n'est par exemple pas encouragé de faire des déductions sur l'existence de couples dans le voisinage des mangeoires. Il est en effet stipulé dans le protocole que les oiseaux qui doivent être comptés sont ceux vus en même temps uniquement. Si le profane, pendant les jours durant lesquels il a consenti a observer, voit un mâle d'une espèce puis une femelle de la même espèce 5 minutes après, alors que le mâle s'est absenté de la mangeoire, il ne doit décompter qu'un seul oiseau, même s'il a la quasi-certitude qu'il y a un couple qui se nourrit à ses mangeoires. Le protocole réduit l'apport du profane à un travail visuel brut, un enregistrement d'occurrences indépendantes.

12. Comme l'explique Roger Slothower : "If I had an observation -this is a map [prend une feuille de papier quadrillé]- I have an observation in this cell and I have an observation in this cell, in this cell, in this cell, but not between them. So how am I going to.... I have to make an interpolation as I say : this is a lot of birds here and a lot of birds here and a few birds here. I can estimate what the entire population looks like. I take this point [ me montre un carreau de la feuille de papier dont on a convenu qu'il contenait des observations positives] and I make a surface. I draw a blanket all over it and connect between the points. That's what interpolation means. It is filling in between the points based on different techniques and the value that you have at the point. Your term ubiquitous makes it. This is an ubiquitus estimate. The term could be misinterpreted because it could mean you want a single number for all area. But it is an ubiquitous surface so every area is represented by this ubiquitous surface. There is an estimate everywhere which may or may not be the exact value of this one that is reported, maybe the mechanism that we use to extrapolate smoothes off the higher points and raises the lower points. And that's a biological scientific decision : how to interpolate. And I use the simplest interpolator that I can find".

13. C'est ainsi qu'est née l'appendice au programme Feederwatch concernant le suivi de l'évolution d'une maladie, la conjonctivite mycoplasmique chez les roselins familiers. Il s'agissait de mieux comprendre l'épidémiologie de cette maladie en grandeur nature, dans un objectif evident de preservation des espèces et de gestion de la population aviaire.

14. Comme John Fitzpatrick le directeur du CLO dit : The idea of Project Feederwatch isn't so much to try to spot a rare bird at a feeder - although that's a bonus- but to count the common, everyday birds that come back every year to the same places" in "Bird-watchers flock to assess the avian situation", USA Today, 16 février 1999.

15. Comme le dit Hochaschka : "The biological justification was that the most common species are the one we get more information for, the fewer 0 s and with more non 0 information, again you have more information to work with, to look for changes in abundance or changes in distribution. So there is a rational reason for being more interested in the common species because those are the ones that we have the most valuable information for. Mostly the rare species are the very unusual ones."

16. Celle de suivre, malgré l'opportunité de formation qu'elle offre au profane, un schéma de communication unilatéral du scientifique au profane, pour la conception des problèmes et le design du dispositif de comptage (Bonney et Dhondt, 1997, 43). 17. Cela explique notamment la conservation des documents papiers, une fois les formularies saisis, au cas où on aurait besoin de retourner à la source. 


\section{RÉSUMÉS}

Le projet américain Feederwatch a pour but de compter les oiseaux aux mangeoires des particuliers de novembre à avril de chaque année depuis 1988. Ce programme s'appuie sur l'outil statistique pour dégager des tendances sur plusieurs années de l'évolution des populations et de leurs aires de distribution, outil nécessité par l'envergure de l'effort de recherche qui concerne chaque année près de 16000 profanes. C'est un cas de vulgarisation scientifique réussie, avec une participation des profanes dans un projet scientifique authentique. Cet article décrit et discute les mécanismes de codage et de traitement statistiques qui assurent à chaque étape l'alignement entre scientifiques et profanes, dans ce que le Laboratoire d'Ornithologie de Cornell, organisateur du projet, appelle une initiative de « science citoyenne ».

Feederwatch program is a project dedicated to counting birds at feeders in the backyard of participants from November to April, since 1988. This program uses statistical means to calculate trends in the evolution of bird populations and areas of distribution, in order to cope, each year, with the huge cover of the program (North American continent) and consequently the huge number of lay people involved (16 000 in 2003). This case documents a successful program in "public understanding of science" oriented toward real science. This paper describes and discuss the various mechanisms aligning scientists and lay people, at each stage of the process of data collecting and processing, in what the Cornell Laboratory of Ornithology calls an example of « citizen science».

\section{INDEX}

Mots-clés : ornithologie, sociologie des sciences, statistique, citoyenneté, sociologie des mouvements sociaux

Keywords : sociology of science, ornithology, statistics, citizenship, sociology of social movements

\section{AUTEUR}

\section{FLORIAN CHARVOLIN}

Florian Charvolin est chargé de recherche au CRESAL de Saint-Etienne. Il a une formation de science politique et de sociologie des sciences, (thèse sous double sceau entre l'Ecole des Mines de Paris et l'IEP de Grenoble), et a travaillé sur l'administration de la nature, du point de vue institutionnel (un livre sur l'invention du domaine public de l'environnement en France) et du mouvement social. C'est à la jonction de ces deux champs qu'il travaille actuellement notamment dans ses recherces sur les rapports entre ornithologie et citoyenneté. 